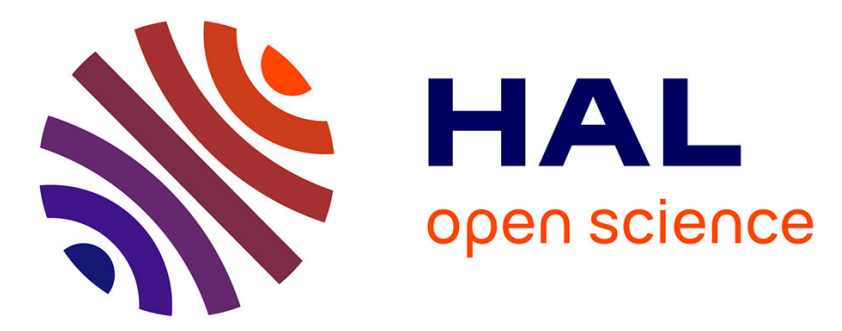

\title{
Fall-Prevention Exergames Using Balance Board Systems
}

Miguel Brito, Rui Nóbrega, João Jacob, Rui Rodrigues, António Coelho

\section{To cite this version:}

Miguel Brito, Rui Nóbrega, João Jacob, Rui Rodrigues, António Coelho. Fall-Prevention Exergames Using Balance Board Systems. 17th IFIP Conference on Human-Computer Interaction (INTERACT), Sep 2019, Paphos, Cyprus. pp.406-426, 10.1007/978-3-030-29384-0_25 . hal-02544594

\section{HAL Id: hal-02544594 https://hal.inria.fr/hal-02544594}

Submitted on 16 Apr 2020

HAL is a multi-disciplinary open access archive for the deposit and dissemination of scientific research documents, whether they are published or not. The documents may come from teaching and research institutions in France or abroad, or from public or private research centers.
L'archive ouverte pluridisciplinaire HAL, est destinée au dépôt et à la diffusion de documents scientifiques de niveau recherche, publiés ou non, émanant des établissements d'enseignement et de recherche français ou étrangers, des laboratoires publics ou privés.

\section{(c)(1)}

Distributed under a Creative Commons Attribution| 4.0 International License 


\title{
Fall-Prevention Exergames using Balance Board Systems
}

\author{
Miguel Brito ${ }^{[0000-0003-4603-2713]}$, Rui Nóbrega ${ }^{1,2[0000-0002-3620-7279]}$, \\ João Jacob ${ }^{1[0000-0003-2078-3375]}$, Rui Rodrigues ${ }^{1,2}$ [0000-0003-4883-1375], and \\ António Coelho ${ }^{1,2[0000-0001-7949-2877]}$ \\ 1 Faculdade de Engenharia da Universidade do Porto, Porto, Portugal \\ ${ }^{2}$ INESC TEC, Instituto de Engenharia de Sistemas e Computadores Tecnologia e \\ Ciência, Porto, Portugal \\ migueldiasbrito@gmail.com, \{ruinobrega, joao.jacob,rui.rodrigues, acoelho\}@fe.up.pt
}

\begin{abstract}
The prevention of falls in older adults is an issue that can only be solved with regular exercises, sometimes with the supervision of therapists. This paper presents a game framework that uses devices such as the Wii Balance Board (WBB) to replicate fall-prevention programs, such as the Otago Exercise Programme. The objective is to increase the adherence of senior users to these exercises, while increasing their autonomy by enabling them to conduct unsupervised games. Two interactive games were developed and tested using the WBB and other available devices such as smartphones. The main contribution of this paper is the proposal of several metrics for exergames for the elderly and the combination of digital games with fall-prevention exercises and automatic balance measures based on Centre of Pressure (COP) tracking.
\end{abstract}

Keywords: Exergames · Fall Prevention · Balance Metrics · Human Computer Interaction.

\section{Introduction}

The increase in quality of healthcare over the past century led to the growth of life expectancy. With this change, age-related problems are more common. One of these problems is related to falls and their potentially serious consequences. As age degrades biological elements that control body balance, falls become more frequent and more severe. Fall prevention programs usually integrate several complementary methods. One of them is doing exercises that improve balance [3] in older adults [1], decreasing fall risk, but also strengthening the body, mitigating fall consequences [8]. However, motivating seniors to do healthy exercises is difficult. Affordable motion sensing devices have been shown to bring motivation as they turn physiotherapy sessions into entertaining and engaging interactive environments. One of those devices, the Nintendo Wii Balance Board System (WBB), uses four pressure sensors that measure the user's center of pressure (COP) which has been proved to be useful in helping the therapists evaluate their patients' balance $[1,9,28]$. 
The main objective of this paper is to propose a methodology for creating and evaluating interactive exergames that provide a solution for motivating older adults towards regular exercise practice [26], as well as automating data collection for healthcare professionals. The usage of multiple sensors and devices can provide the system with input both for exergames and for balance metrics. A combination of game development techniques and fall-prevention exercises may not only motivate older adults as it combines the pleasure of playing video games with the need to promote balance [27], but also provide physiotherapists with data from remote game play sessions.

To test the proposed methodology and metrics, two interactive exergames were developed. These games require the user to perform exercises that are part of the main fall-prevention programs [8], such as the Otago Exercise Programme or the Fitness and Mobility Exercise (FAME), and balance assessment scales as well, such as the Berg Balance Scale [22]. While the senior plays the game, the system uses the COP and compute balance assessment metrics used by physiotherapists, namely the COP's mean velocity and total oscillation. This data can be further processed and COP's path, amplitude-over-time and frequency maps can be generated.

\section{Related Work}

The scope of this work involves exergames in health [31,17]. Digital games can provide enjoyment to people, defined here as an individual's positive response towards technology and its context. Experiencing enjoyment is the main reason why people play games [23]. A difficult game for a novice player can create anxiety making him quit, while a very easy game can cause boredom[18, 19]. Increasing challenges' difficulty as the player progresses and gains skill is then important [24]. This way, fun can be achieved throughout the whole game [7]. Game style factors can be determinant when providing enjoyment to players. Fantasy inclusion, narrative, avatar resemblance and player's identification with it, other playable characters, sound and music effects, high quality realistic graphics, use of humor, character development over time and game medium duration (few days or weeks) affects the way players feel about the game [23, 24]. Psycho-social characteristics, which are player-dependent, also affect the way a person enjoys a game. The most important player's traits are sensation seeking, self-forgetfulness, desire of being in control, self-efficacy and need of satisfaction (mood repair or recovery of an experience) [23]. Games that cause guilt, e. g. violent games for some players, are not effective to provide enjoyment [23]. Several authors studied enjoyment in games such as Mellecker et al. [24], Zaczynski et al. [38] and Nawaz et al. [27].

\subsection{Therapy-Oriented Exergames}

Deutsch et al. [11] made a first approach to the use of Nintendo Wii console in physical rehabilitation. An adolescent student with spastic diplegia cerebral 
palsy was subjected to eleven sessions during four weeks where he would play games from a set of four Wii games and given the liberty to play each game for as long as he wanted. The therapist observed that while playing the digital games, the patient did not feel he was in therapy and did more effort in the exercises.

Exergames to improve balance in older adults is a topic that was explored in Agmon et al. [1]. In this work it was asserted that limited supervised balance training at home was safe and feasible. Furthermore, several other games studied the use of exergames in older adults [3] and in unsupervised scenarios [28].

Lange et al. [21] developed an exergame for neurological injury rehabilitation using the WBB. More recent works indicate important factors to be considered to develop motivational rehabilitation exergames. Geurts et al. in [14] concluded that it was important to be possible to calibrate the exergames according to the patients' needs and capabilities. Uzor and Baillie [36] developed five exergames for seniors, as well as wireless inertial sensors to interact with them. These sensors detect nine movements, four of those are used in each game, which are: sit-tostand, side steps, marching, knee bends.

Vines et al. [37] elaborated a critical analysis of over 30 years of HCI research for the elderly with the goal of finding its main concerns and give advices for future studies. Elderly adults enjoy interacting with Nintendo Wii controllers and find them useful for therapy despite technology and physical limitations [6]. Nielsen and Störring [29] also proposed a method to create an interface with hand gestures.

Recently, Clark et al. shown that the WBB can be used in clinics to compute quantitative balance measures, namely center of pressure, path length and velocity, with high correlation with precise commercial force plates that are the gold standard in quantitative balance measurement [9]. González et al. developed a Center of Mass estimation system which uses the Kinect and the WBB using the statically equivalent serial chain method [15]. The solution is a portable and affordable alternative to other systems despite its limitations. Improper light, loose fitting clothes and large objects surrounding the user interferes Kinect's readings while WBB area limits the number of poses the user can make [15]. Other balance therapy projects include Kennedy et al. [20] and Ayoade and Baillie [2].

\subsection{Balance Rehabilitation Overview}

Balance maintenance requires coordination from sensory, neurological and musculoskeletal systems [22]. Commercial force plates are used to compute a person's COP while standing. These devices can be used to identify older adults with higher fall risk even when there is no evidence of a balance impairment [30]. With the COP values over some time, one to two minutes, several variables can be determined for balance assessment [12]. COP can be measured in two axis: backward and forward or anterior-posterior direction (AP), and side-to-side or medial-lateral direction (ML). The most useful metrics are the stabilogram's 
bandwidth and the COP path, medium velocity, oscillation area and total displacement [12]. Higher COP bandwidth, alongside inability to tandem standing (stand with a foot in front of the other with the front foot's heel touching other's toe) and higher body mass index, indicates higher probability of a fall occurrence due to intrinsic factors [30]. The COP test should be done two to four times as too many repetitions may cause fatigue and learning, which can result in a wrong analysis [12].

Laboratory testing of balance using treadmills or sway platforms is impractical in most clinics [32]. Therapists nowadays use subjective scales thoughtfully tested based on functional balance tests. Functional balance tests are exercises based on basic movements such as getting up from a chair, standing or picking up objects. One of those tests is the Time Up and Go test (TUG). The TUG consists in getting up from an arm chair, walk three meters, turn, come back and sit in the chair [32]. Evaluation is given relative to the time the patient takes to complete the task. Patients who take less than twenty seconds were shown to be independent for basic mobility activities whereas those who took more than thirty had difficulties getting up of bed, sitting and getting up of a toilet and getting in and off a tub [32]. TUG is a reliable test to assess patient's balance, gait manoeuvre and functional abilities and track physical mobility deterioration [32]. However, TUG does not give enough information to detail the source of balance impairments [22].

Other tests include the Berg Balance Scale (BBS) [22,1], the Fullerton Advanced Balance Scale (FAB) [33], the Brunel Balance Assessment (BBA) [35] and the Functional Independence Measure (FIM) [20]. Different exercise programs have been created and successfully tested regarding fall-prevention. In New Zealand, Campbell and Robertson's Otago Exercise Programme has decreased fall frequency by one third in four trial tests and improved senior's self-confidence for daily activities [8].

\section{Proposed Solution}

The main contribution of this work is that it provides a methodology for integrating known physiotherapists' exercises in exergames that can be played with relative autonomy while providing feedback to the therapists. This methodology focuses on the definition of a set of metrics to ascertain balance as well as sensors and devices to collect data.

To validate this methodology, two exergames, fit to be played by elderly people and with specific exercise movements to prevent falls, are described and tested. The goal is to implement balance assessment with automatic metrics used by physiotherapists to provide a mean to monitor the player's evolution while keeping subjects motivated.

The designed exergames are digital games where the player must perform one or multiple exercises to control a character and solve problems. Problem-solving levels and game rewards are important as they were shown to be effective to motivate patients for therapy sessions [5]. The exercises were chosen from main 
used fall-prevention exercise programs to hopefully improve older adults balance and decrease fall risk [8].

The methodology consists of the following steps: (1) choose exercises, (2) choose devices, (3) choose metrics, (4) create exergame and (5) evaluate with therapists.

\section{Balance Metrics}

The main input device chosen was the Nintendo Wii Balance Board (WBB). The WBB was developed and commercialized by Nintendo initially as a controller for some WiiFit mini-games. In these games, the WBB tracks the player's Centre of Pressure (COP) whose position has to be controlled in order to progress.

Data from the WBB is sent via bluetooth connections and includes the pressure measured by each sensor and total. This way, COP value can be computed using equation 1 . Let $i=1 . .4$ be an identifier for each sensor (front left, front right, rear left, rear right), $P_{i}$ the position of sensor $i$ and $W_{i}$ the weight read by sensor $i$. Then:

$$
C O P(M L, A P)=\sum_{i=1}^{4} \frac{W_{i} * P_{i}(M L, A P)}{\sum_{i=1}^{4} W_{i}}
$$

Manipulating the position of each sensor in the equation according to a rotation from the WBB original position will give a correct COP in Medial-lateral (ML) and Anterior-Posterior (AP) directions. Tracking the user's COP, several movements or exercises can be detected or measured.

One goal for this project was to provide physiotherapists or care-takers a mean to monitor a senior's balance evolution. Equation 1 allows COP computation with data from the WBB. However COP alone is not useful for balance assessment [12]. Duarte and Freitas [12] indicated that the best COP related metrics for balance assessment were COP's mean velocity, total oscillation, path chart and displacement (amplitude) over time.

\section{Game Design and Implementation}

Following the methodology, after identifying certain exercises and considering the balance metrics to be collected, two exergames were designed.

For both games we describe the design and implementation steps. Both games have a small calibration stage and a tutorial as seen in Figure 1, followed by the game logic. They are based on a city environment, divided in several streets. The city has several objects, such as cars and boxes that the player has to avoid. This provides a setting that is familiar to all players.

The games display information to the player in order to help him. As previously mentioned, tutorial messages appear to the player in the beginning of 


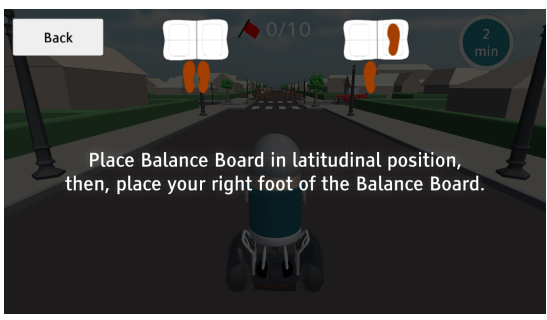

(a) Segway Stroll

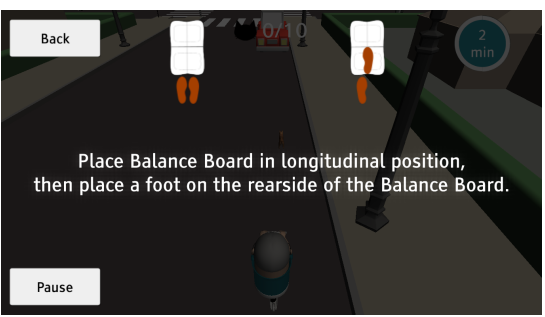

(b) Scooter Chase

Fig. 1. Tutorial messages for Segway Stroll and Scooter Chase.

the game, either to instruct the player to calibrate the devices or to explain the game's logic and mechanics, as seen in Figure 1.

The next sections briefly describe the features of each game.

\subsection{Segway Stroll - Description}

In this game, the player has to drive a virtual Segway (http://www.Segway.com). To interact, the user has to stand in the WBB and perform forward reaching movements or weight shifts. These movements can be read by the WBB alone. However, other sensors can be added in order to force the user to do them correctly. Other movements can be included by introducing obstacles in the levels. Figure 2 is a sketch of the idea for this game.

In the game the player is leaving home to try out the newly bought Segway and takes a stroll in the town with the objective of reaching the end of a path. The user has to forward reach in order to move, stand to stop and perform weight shift to turn.

Exercise: To perform a forward reach, the player must stand with feet shoulder-width apart. Then, raise one arm to the shoulder height and lean forward as far as possible. To finish the forward reach the player must return to the initial position. In this exercise the player should alternate the raising of their arms. The initial position for forward reaching and weight shifts are the same. To perform a weight shift, the player should bend one of the legs placing more weight over that leg. Weight shifts should be done for each leg, alternately. First levels have shorter paths and require fewer movements and have shorter game play sessions. Higher levels have more complex paths with more demanding exercises.

Benefits: These game's movements help seniors train balance control when their centre of gravity changes [25]. Forward reaching improves the limits of forward stability [25]. Weight shifts are present in FAME [13] and BBA [35], forward reaching is present in FAME, Melo's program [25], BBA and BBS [4] and standing is part of BBS and BBA and provides quantitative data for balance assessment [9].

Evaluation: Sensors used to read the player's movements provide useful data for analysis. The WBB can track changes to the player's centre of pressure in all 


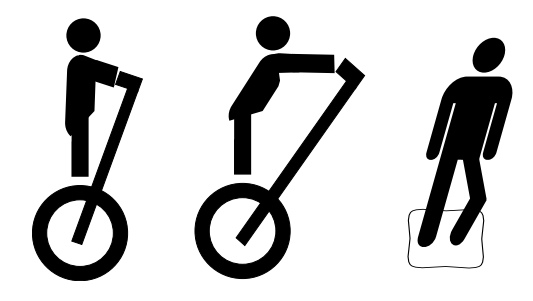

Fig. 2. Segway Stroll sketch. The player has to perform a forward reaching to accelerate and weight shifts to turn.
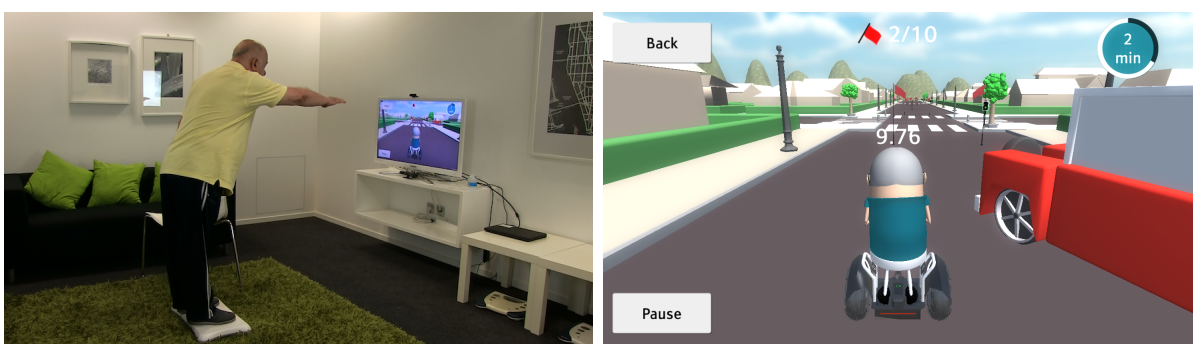

Fig. 3. Senior performing a forward reach movement while playing Segway Stroll.

movements. An external camera and body tracking software can be used to force the player to do the movements correctly, in terms of body pose. Smartphones or smartwatches can also be used when forward reaching in the player's hand or wrist.

Evaluation measures taken from this exercise are: the number of repetitions and duration of each movement, the amplitude of forward reaching and weight shifts and the COP while the player is standing which can be used to compute other metrics useful for balance assessment, e. g. COP path and COP velocity [9].

This game presents an interactive way to assess balance, as the movements tracked are part of several measures. While useful for assessment, these exercises aim to promote balance when done regularly and consequently, decrease fall risk.

\subsection{Segway Stroll - Implementation}

In this game the main goal is to follow a path given by the system. The player uses the WBB with two different exercises to fully control the character as seen in Figure 2. A senior playing this game can be seen in Figure 3.

A level is comprised of ten checkpoints, each represented as a pair of flags placed on the road. The player has to pass between the two flags to complete the checkpoint and earn points. If the player surpasses a checkpoint without passing between the flags, it will disappear and the player does not get a second 
chance to earn points in it. A time limit of 150 -seconds exists for the game to be completed.

Game Interface The game is placed in a city with several static elements, except for the cars that come from different directions. This game's main goal is to pass between the flags of each checkpoint. The player has a time limit to reach the next checkpoint and the remaining number of seconds appears in the screen. The number of checkpoints successfully surpassed and the total of checkpoints are displayed on the top of the screen. Lastly, traffic lights are present as well as other obstacles to the player. The game interface can be seen in Figure 3.

Calibration and instruction messages are shown to the player in the beginning of the game. A message display can be seen in figure 1. The same method is also used to warn the player that he was not supposed to advance when the traffic light is red or a car is crossing the street.

The score screen presents the number of surpassed checkpoints, the number of obstacle hits, the number of times the player mistakenly advanced through the street (i.e., crossed the street with red light on or when a car was crossing in front) and the total COP's amplitude in the AP direction as an evaluation metric.

Basic Gameplay and Controls To accelerate, the user performs a forward reaching exercise. Forward reaching is detected by the system using the readings from the WBB. The Segway will accelerate more if the COP further moves forward in the AP axis, motivating the player to increase the amplitude of this exercise. A chair is recommended to be used as support to avoid injuries from the player falling forward. The senior playing this game on Figure 3 is performing this exercise to accelerate the Segway.

The second interaction is turning the Segway. This can be made by weight shifting to the desired side while forward reaching. This mechanic was implemented to add another degree of interaction to the game. The WBB sensitivity to turn is very high in order to make this interaction effortless.

Lastly, to brake the Segway, the player must perform a toe raise. This exercise was added as it is used in fall-prevention exercise programs. The Segway will lose velocity gradually when not accelerating, but braking was added to provide the ability to perform sudden stops which are important to avoid traps. Once again, a support should be present in order to avoid the player falling backward as the WBB front may rise when the player performs a toe raise. This exercise can be seen on Figure 4.

Exercise Analysis In order to evaluate the forward reaching exercise, therapists usually measure the maximum distance the patients can reach with their arm. This analysis is hard to assess using the WBB, and further sensors should be added for better exercise tracking, as previously suggested. Nevertheless, the system collects the maximum distance the COP reaches in the AP direction and presents it to the user in the score screen. 


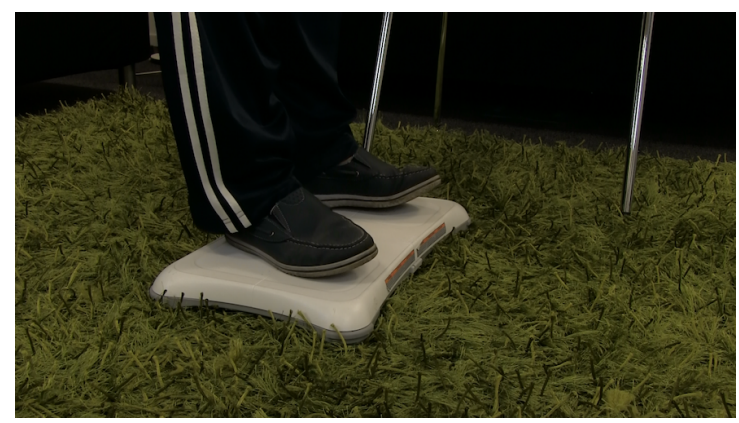

Fig. 4. Senior performing a toe raise movement on the WBB required to play Segway Stroll.

The purpose of traps in this game is to force the player to stand still for a few seconds. This way, quantitative metrics can be taken for further analysis as indicated by Duarte and Freitas [12]. However, the traps are activated for 10 seconds, at most, instead of the minimum of 30 seconds required for a correct assessment according to Duarte and Freitas [12]. This decision was made because seniors could get bored with the game if they had to stand for an extended period of time, thus failing to motivate them to play. Figure 5 presents a COP's path chart, a COP's frequency map and an amplitude-over-time chart. All the examples were taken from a participant gameplay during test phase, which will be described in the Evaluation section. These metrics could provide data to physiotherapists so they can perform a balance assessment on their patients.

\subsection{Scooter Chase - Description}

In this game, the player has to do a tandem stand, i.e., stand with a foot in front of the other, in order to balance while riding a scooter. The movement performance is read by the WBB and transmitted to the game. A smartphone is used as a steering wheel.

The player notices something is missing: the cat ran away. The player follows the cat, but it is too fast. The player sees the grandson's scooter, gets on it and accelerates to catch the cat. Tandem standing is needed to keep balance and avoid falling from the scooter. The main goal of this game is to catch the cat and return without falling.

Exercise: In order to perform a tandem standing, the player must place a foot directly in front of the other and hold for some seconds. For the movement to be read by the $\mathrm{WBB}$, the player should turn it $90^{\circ}$ from its usual position and place one foot on the rear side and the other in the front side of the board, as seen in Figure 1 b). In higher levels, the scooter's sensitivity to COP changes and the time needed for the player to stand in a tandem position increases. 

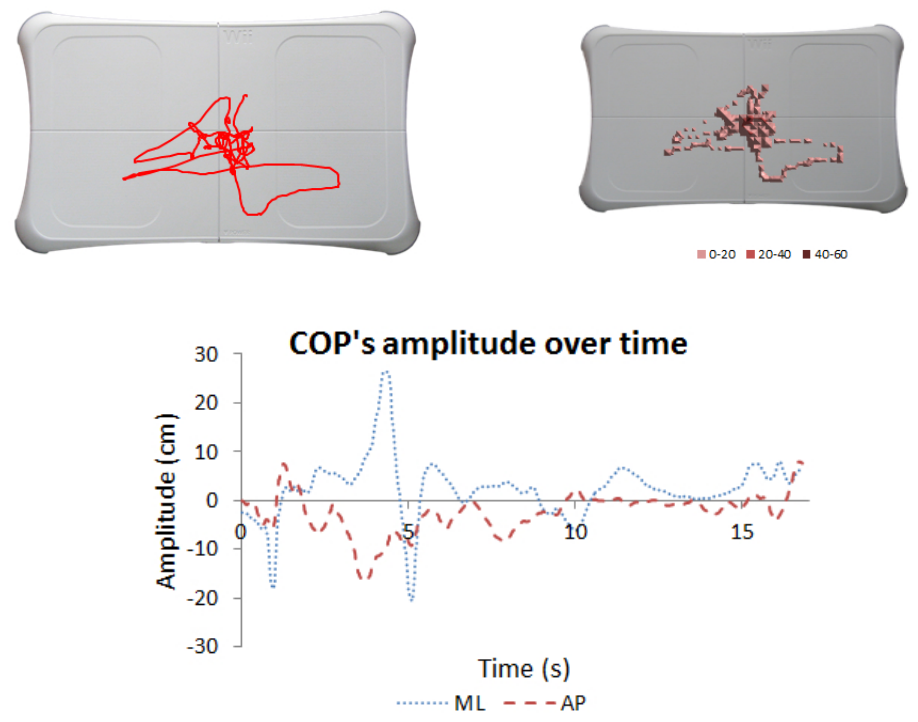

Fig. 5. COP's path chart on the left, COP's frequency map on the center and COP's amplitude-over-time chart on the right while standing, taken from Segway Stroll.

Benefits: Heel-to-toe standing is indicated in Otago [8] and FAME [13] programs as a means to promote older adults' balance. In addition, tandem standing is also used as a balance measure in the BBS [4].

Evaluation: The WBB provides information about the tandem standing. These readings can be used to determine the number of repetitions and the duration of each standing. COP path and sway in this exercise may be useful to assess balance as well.

Tandem standing promotes balance and consequently, decreases fall risk. At the same time, it can also be used to assess balance.

\subsection{Scooter Chase - Implementation}

To simulate a scooter's base, the system relies on a WBB, rotated $90^{\circ}$ from its normal position, and a smartphone to simulate the handlebar. This game can be used to promote balance improvements and to assess it as well. A senior playing this game can be seen in figure 6 . The main goal is to catch the cat. However, it is not enough to catch it one time, as it runs away again several times. The player has a 150 seconds time limit for reaching the cat on ten occasions.

To achieve a better result, the player should avoid obstacles in the scenario such as cars and streetlights. Balance performance is also considered when computing the final result to motivate the player to sustain the tandem standing longer and tremble less. 


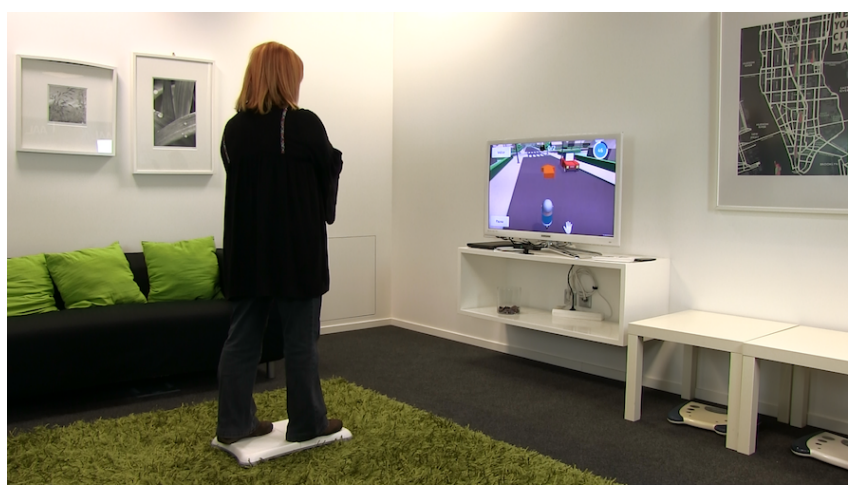

Fig. 6. A senior playing Scooter Chase.

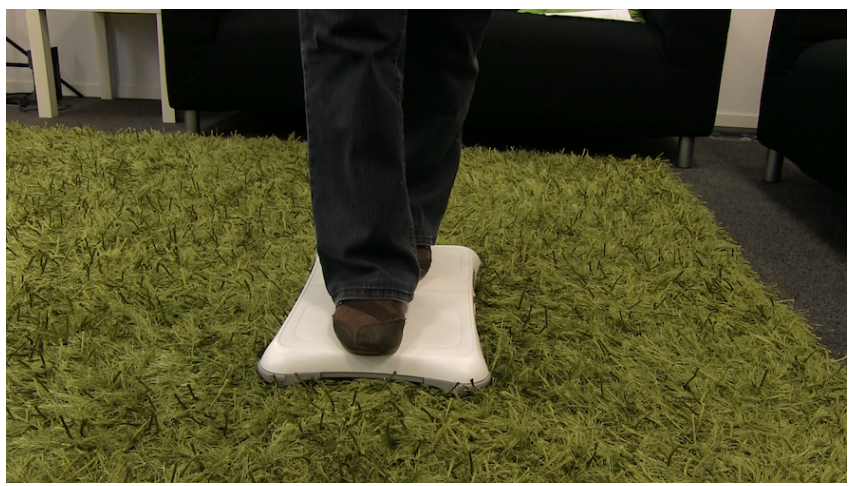

Fig. 7. Heel-toe standing on top of the WBB required to play Scooter Chase.

Basic Gameplay and Controls There are two interactions possible in Scooter Chase: move and turn.

The main development goal was to use a tandem standing exercise as an interaction to move the player. An algorithm was written to detect when the player is doing the exercise and when it does, the game character moves at constant speed, stopping when the player leaves the position. Stopping can also be used to figure what to do next. A player performing the tandem stance in order to play this game can be seen in figure 7 .

The possibility of turning was added to make the game more dynamic and attractive, enabling the cat to make curvilinear trajectories and adding obstacles in the path which the cat can jump, as well as requiring the player to coordinate the movement of the handlebar with the stance. The first approach to this interaction was to use COP's sway in the medial-lateral direction using arms wide open rotations to cause it. However, this decision did not affect the COP coordi- 


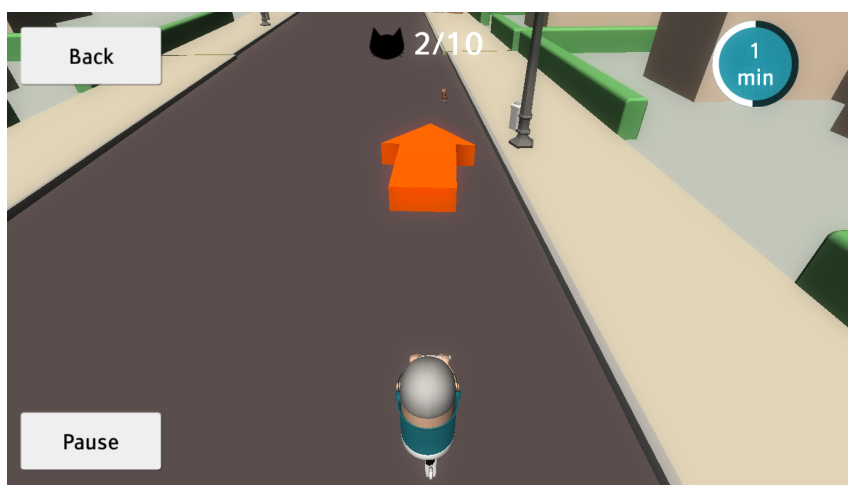

Fig. 8. Scooter Chase screenshot.

nates: that arm rotation barely affected COP because the human body tends to compensate these movements with the feet, causing the COP's to actually move in the unintended direction in most cases. Ultimately, smartphone interaction was added, as an handlebar, to surpass those difficulties, using changes in its three-dimensional orientation to rotate the character. Adding a second device was more effective than using the WBB to turn while tandem standing.

This game has four difficulty levels where the path the cat runs is different and has more obstacles for the player. The cat and scooter's speed and handlebar's sensibility increase in higher levels. The first level has a small tutorial to explain to the players the mechanics and the logic.

When the player catches the cat for the tenth time or the time limit is surpassed, the game ends and a score is given and displayed to the player. This score takes into account the number of times the cat was caught. The score screen also shows the number of times the player hit an obstacle, the total time standing in the tandem stance and the balance performance according to the Tandem Stance Test [16] described in the exercise analysis.

Game Interface Scooter Chase displays several information to the player in order to assist. During gameplay, the player can see in the top of the screen the total number of stops the cat does during the level, as well as the number of times it was already caught. A clock is also present to indicate the remaining time the player has to finish the game at the top right corner. An arrow will appear above the character if the cat is far way to help the player locate it. A game's screenshot can be seen in figure 8 .

As stated above, tutorial messages will appear to the player in the beginning of the game, either to instruct the player to calibrate the devices or to explain the game's logic and mechanics as seen in figure 1 . The score screen, seen at the end of the game, presents scores to the user, which are the number of times the 

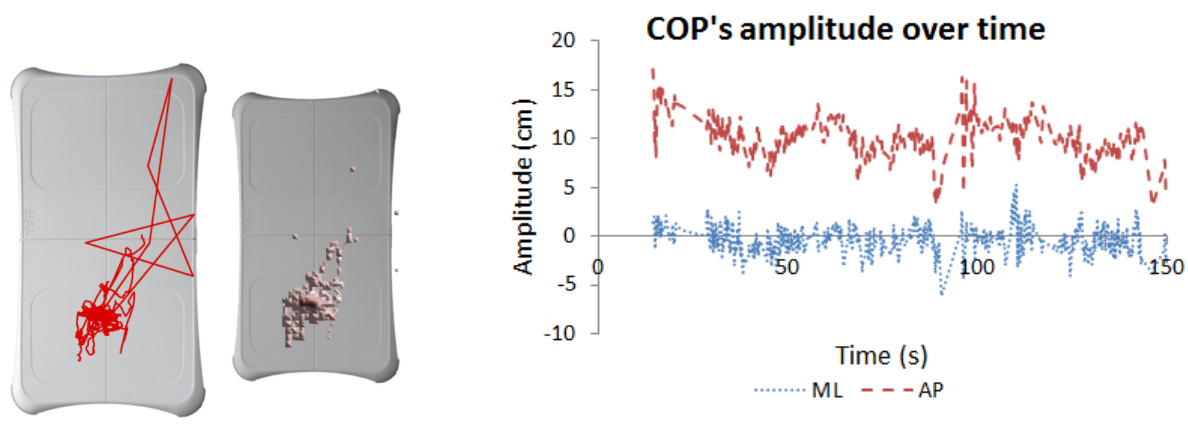

Fig. 9. COP's path chart on the left, COP's frequency map on the center and COP's amplitude-over-time, all taken while performing a tandem stance in Scooter Chase.

cat was caught, the number of times the player hit an obstacle, the total time standing in the tandem stance and Tandem Stance Test (TST) result.

Exercise Analysis The first balance assessment metric present in this game is the Tandem Stance Test (TST) [16]. This test is clearly appropriate to use in this game as it takes into consideration the maximum time a patient can maintain a heel-toe standing pose. The result from this test can be low, if the maximum time is between 0 and $9 \mathrm{~s}$, medium if between $10 \mathrm{~s}$ and $29 \mathrm{~s}$, or high otherwise. The terminology was changed to weak, fair and good to inform the players better about the assessment. A classification of excellent was added if the player can maintain the pose through all the game.

In addition, the COP's mean velocity and total oscillation are computed and recorded in a file. This file also contains the COP tracking needed to generate the COP's path, amplitude-over-time and frequency map. On figure 9 it is presented, on the left, an example of a COP's path chart, on the center a COP's frequency map and on the right is a COP's amplitude-over-time chart, all taken from one participant's tryout. These metrics could be useful for a balance assessment from a physiotherapist. This approach may be a feasable way to regularly evaluate patients remotely through the use of exergames.

\section{Evaluation}

The test focused on the system's usability and satisfaction, and in field-testing a set of proposed metrics. The volunteers experimented the final versions of Scooter Chase and Segway Stroll. This test phase counted with eleven participants. These volunteers were seniors who lived independently with ages between sixty-four and eighty years old $(\overline{\mathrm{x}}=72.09 \pm 5.75)$. Six participants were of the female gender. 


\subsection{Methods}

Before interacting with the developed exergames, the volunteers were asked to perform the Time Up and Go (TUG) test. Then, the participants would experiment the system following the instructions of a mediator. The mediator instructed the volunteers as follows:

1. Perform the TUG test.a) Begin seated. b) When the mediator says "go", the volunteer should get up, walk three meters, return and sit again where the participant was. c) The three meters distance is pointed out by the mediator. d) The mediator must start a stopwatch when saying "go", stop when the participant sits and write the time.

2. Play the Scooter Chase game. a) Read the calibration instructions and interact accordingly. b) Read the game instructions and play the game. c) If the player does the calibration wrong or does not understand something, the game should be restarted. d) If the player has not understood, the mediator should explain or demonstrate the instructions.

3. Play the Segway Stroll game. a) Read the calibration instructions and interact accordingly. b) Read the game instructions and play the game. c) If the player does the calibration wrong or does not understand something, the game should be restarted. d) If the player has not understood, the mediator should explain or demonstrate the instructions.

4. Answer the questions on the questionnaire.

This protocol provides a balance test result for comparison with the automatic system analysis to evaluate if the system assessment was consistent. The questionnaire has questions about physical exercise habits and motivation to play these games. All the measured metrics are described in detail in the next section.

\subsection{Measured Metrics}

As stated, with this work we aim for solutions that can motivate older adults. Knowing that technological barriers and complex mechanics can hamper the perception of the games, and hence their acceptance, we chose a set of metrics to assess these issues in the case of our proposed games. Metrics M1, M2 and M3 cover the technological expertise level, needs for explanation, and the games difficulty perception (Table 1).

The second set of metrics (M4, M5 and M6) focuses on the automation of data collection for healthcare professionals (another of the goals of this work). Therefore they cover data obtained automatically by the system regarding COP (described in section 4), TST and TUG (supported by the literature).

For the Segway Stroll, the system tracks the COP when the player is standing still for more than 10 seconds. It records all COP readings and respective time during the tracking in order to draw the COP's path, COP amplitude over time in AP and ML directions and a frequency map. In addition, it records the 
Table 1. Metrics summary.

\begin{tabular}{llll}
\hline ID & Metric & Source & \multicolumn{1}{c}{ Description } \\
\hline \hline M1 & Technological ex- Questionnaire Number of devices regularly used \\
& pertise level & (phone,PC,tablet) $[0,3]$. \\
\hline M2 & $\begin{array}{l}\text { Explanation level Annotation } \\
\text { required }\end{array}$ & $\begin{array}{l}\text { Explanation required from the mediator }[1,5] \\
\text { based on annotations (see Table 2). }\end{array}$ \\
\hline M3 & $\begin{array}{l}\text { Games' difficulty Questionnaire } \\
\text { perception }\end{array}$ & $\begin{array}{l}\text { Median of Likert scores [1,5] of questions (see } \\
\text { Table 3). }\end{array}$ \\
\hline M4 & $\begin{array}{l}\text { COP's mean } \\
\text { locity }\end{array}$ & ve- System & $\begin{array}{l}\text { COP's mean velocity while performing a tan- } \\
\text { dem or standing stance measured by the sys- }\end{array}$ \\
& & tem. \\
\hline M5 & TST & System & Maximum time maintaining a tandem stance. \\
\hline M6 & TUG & System & Results from TUG test. \\
\hline
\end{tabular}

COP's total mean velocity, total oscillation, standard deviation and variance. A summary of all metrics used in the tests is described on Table 1.

For the Scooter Chase analysis this data was taken from the system: total time in tandem stance, maximum time in tandem stance (which is used by the system to evaluate the player's balance using the TST [16]), COP's mean velocity and total oscillation through the game, the number of repetitions, for each of them, the standard deviation and the variance in $\mathrm{AP}$ and $\mathrm{ML}$ direction and lastly, COP read while in the stance and the time it was taken, used to draw the COP's path, COP frequency map and COP's amplitude over time.

\subsection{Results and Analysis}

This section presents the main results of the tests. The technological expertise (M1) for this population was characterised in terms of regular use of smartphones, tablets and computers or laptops. Three participants said they do not use any of the devices regularly, two stated that they used the smartphone and two other said they used smartphones and computers. From the remaining participants, one said he uses only computers, another uses only the tablet, the third uses the smartphone and the tablet and the last said he uses all the devices regularly. As such, this group had disparate technological habits.

The explanation level required (M2) to play the exergames is detailed in Table 2 while median values of the answers to the games' difficulty questionnaire (M3) can be seen in Table 3. The average of the TUG result and automatic retrieved answers (Metrics M4 to M6) are presented in table 4.

The annotations (M2) seen in Table 2 use a 5 point Likert-scale. With these annotations we could see that most users could understand well the WBB interface, namely in Scooter Chase users did understand how to move with the heel-toe standing $(M=5)$ and how to move using a forward reach in Segway Stroll $(M=5)$. Most users were able to understand the calibration system in both games without much explanation. The usage of the smartphone to steer 
Table 2. Explanation level required - Mediator Annotations, Metric M2 (Medians and Interquartile Range), some annotations were only made for one application

\begin{tabular}{lccc|cccc}
\hline Q & \multicolumn{3}{c}{ Scooter Chase } & \multicolumn{3}{c}{ Segway Stroll } \\
\hline \hline & M & Q3 & Q1 & M & Q3 & Q1 \\
\hline 1 How to move & 5 & 0 & 0 & 5 & 0 & 0 \\
2 WBB calibration & 5 & 0 & 0 & 5 & 0 & 0 \\
3 Smartphone calibration & 5 & 0 & -2 & & & \\
4 How to steer & 3 & 2 & -1 & 5 & 0 & -4 \\
5 Must catch the cat & 2 & 2.5 & -1 & & & \\
6 How to brake & & & & 3 & 0 & 0 \\
7 Must avoid obstacles & 4 & 1 & -3 & 5 & 0 & -4 \\
\hline
\end{tabular}

Table 3. Questionnaire Metric M3 (Medians and Interquartile Range)

\begin{tabular}{lccc}
\hline$\# \quad$ Question & M & Q3 & Q1 \\
\hline \hline 1 I practice physical exercise regularly & 5 & 0 & -1 \\
2 I enjoyed playing Scooter Chase & 4 & 1 & -0.5 \\
3 I enjoyed playing Scooter Stroll & 4 & 1 & 0 \\
4 I would like to play these games regularly. & 5 & 0 & -0.5 \\
5 I felt I was doing physical exercise/effort. & 4 & 1 & 0 \\
6 These games motivate physical exercise practice. & 5 & 0 & -0.5 \\
7 Device calibration was simple & 3 & 0.5 & 0 \\
8 Controlling the vehicles was simple & 3 & 0 & -1 \\
9 Objective fulfilment was simple & 3 & 0 & -1 \\
\hline
\end{tabular}

the Scooter was not very successful $(M=3)$. Some interface elements such as the arrow to track the cat were not obvious $(M=1)$.

The data was retrieved from the system to compare with the results of the TUG test. Few participants had their standing stance assessed by the system, as seen in the last columns of Table 4. First, almost all participants thought they had to raise their calves. One participant after corrected stated "in fact, in the image the figure is also raising the toes". The second reason was that they had difficulties in doing an acceleration-brake movement and were not being able to surpass the first trap before the time ran out. In the future a more thorough exemplification of the exercise and additional time to complete the exercise can help prevent these issues.

All participants scored less than $20 \mathrm{~s}$ in the TUG test as expected for older adults who live independently [32]. With the exception of one participant, all seniors scored more than $30 s$ in the TST which indicates the same lack of balance impairments [16]. As an exception one participant stopped the stance many times to stop the scooter and figure what to do and not because he could not maintain the position. This means that some results may be directly influenced by game design and the learning capacity of the players and not due to physical or motor impairments. This should be further explored in the future. 
Table 4. Automatic measures for each participant (Metrics M4, M5 and M6).

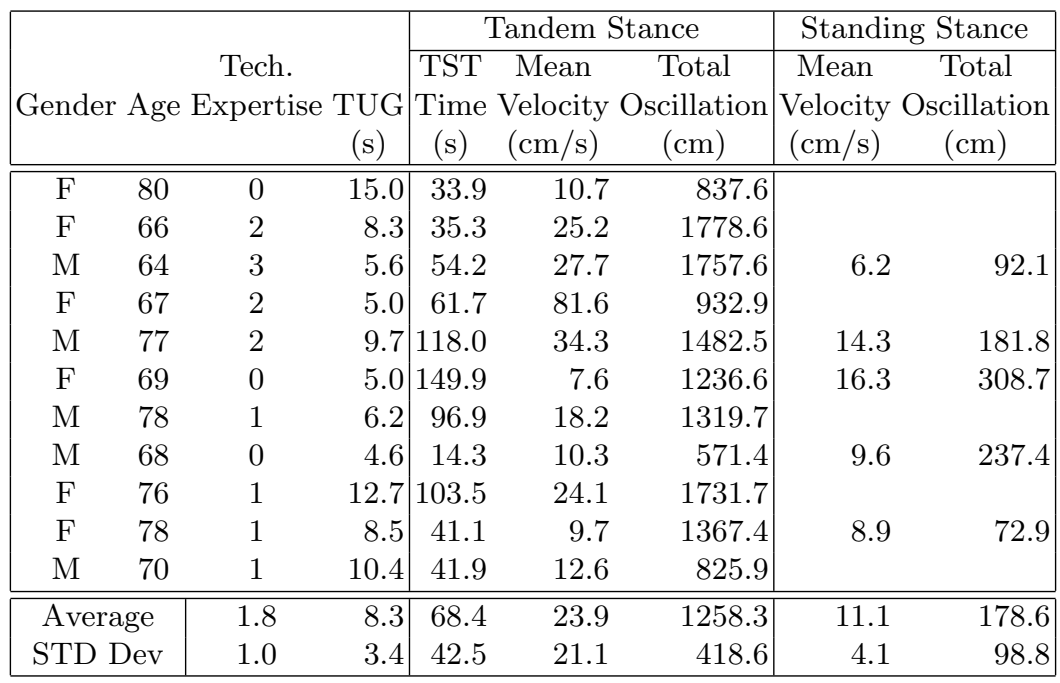

In the questionnaire (M3) (seen in Table 3), all volunteers, except one, said in the first question that they practice exercise regularly. Some even commented what exercises they usually do, such as gymnastics or tai-chi. The results of TUG and TST may be explained by these practices. Despite the obvious difficulties in playing these games, most seniors claimed they liked Scooter Chase, and also enjoyed Segway Stroll. All the seniors said they would like to play these games regularly and by doing so, it would motivate them to do exercise. Most seniors also expressed they felt they were doing physical exercise or effort. The last three questions were focused on the usability and received less favourable answers, which was expected given the level of explanation required for playing.

\subsection{Discussion}

The tests showcased the importance of using a set of metrics to systematically collect and measure performance of older adults in an exergame scenario.The participants claimed they enjoyed playing the games (Metric M3) and they would like to do it regularly, showing motivation towards regular exercise practice.

In this small sample, age analysis suggests users with age below 70 years old understand better the games, but balance is similar across all ages. As almost all the participants exercise regularly, this result is not surprising. Quantitative balance comparison between metrics did not find any relevant relations, as the metrics used are more related to finding balance impairments than quantifying balance in healthier individuals. Further testing these measures with physiotherapists may help reach additional conclusions, which can culminate in the revision of these proposed metrics. Still, qualitative measures between TUG and 
TST did obtain similar results, which indicates that this metric could be used to supervise the players.

TST, COP's mean velocity, total oscillation, path, amplitude-over-time and frequency maps (Metrics M4 to M6) can give a mean for physiotherapists and care-takers to supervise the elderly adults who play the games, thus it can be practicable. Frequent exercise has benefits, so it is expected that regular exergaming sessions would have too. The proposed exergames have to be further studied to reveal their practicability to monitor patients' balance and benefits related to fall prevention. In Segway Stroll the game instructions were considered good, with the exception of the toe raise problem. The forward reaching detection algorithm had some problems as some players had difficulty to move the character. This happened in the preliminary tests during implementation phase. Another difficulty was to avoid traps. As this was the first interaction with the system, players were not expecting the traps and did not stop in time. Then, the participants had problems doing a quick acceleration and braking immediately.

\section{Conclusion}

Digital games for rehabilitation are an important topic of research nowadays and multiple works have presented positive results in using motion sensing devices in therapy despite being originally created for entertainment $[1,28,7]$. Exergaming has been shown to have potential to improve traditional therapy exercises and fall-prevention exercise programs in the works of Deutsch et al. [11], Geurts et al. [14], Uzor and Baillie [36], Davies et al. [10], Santos et al. [34] and others. Our methodology combines this research with a proposed set of balance metrics and therapeutical exercises, such as those proposed by Clark et al. [9] and Kennedy et al. [20] to allow for supervised domestic exergames.

We have proposed a set of balance metrics to be computed while the user plays the games. These proposed metrics for evaluating balance exergames seem to be relevant but further studies are required in the future to refine them.

All participants in the user studies stated they enjoyed playing the games, would like to do it regularly and that the games motivate physical exercise practice. This indicates that home exercise through the use of exergames can be motivating although further studies are needed. Nevertheless there is still room for improvements taking into account the suggestions and difficulties observed in the user studies.

\section{Acknowledgments}

We would like to thank Fraunhofer Portugal for their support. This work is financed by the ERDF European Regional Development Fund through the Operational Programme for Competitiveness and Internationalisation COMPETE 2020 Programme and by National Funds through the Portuguese funding agency, FCT Fundação para a Ciência e a Tecnologia within project POCI-01-0145FEDER-030740 PTDC/CCI-COM/30740/2017. 


\section{References}

1. Agmon, M., Perry, C.K., Phelan, E., Demiris, G., Nguyen, H.Q.: A pilot study of Wii Fit exergames to improve balance in older adults. Journal of Geriatric Physical Therapy 34(4), 161-167 (2011). https://doi.org/10.1519/JPT.0b013e3182191d98

2. Ayoade, M., Baillie, L.: A novel knee rehabilitation system for the home. Proceedings of the 32nd annual ACM conference on Human factors in computing systems - CHI '14 pp. 2521-2530 (2014). https://doi.org/10.1145/2556288.2557353

3. Bateni, H.: Changes in balance in older adults based on use of physical therapy vs the Wii Fit gaming system: A preliminary study. Physiotherapy (United Kingdom) 98(3), 211-216 (2012). https://doi.org/10.1016/j.physio.2011.02.004

4. Berg, K., Wood-Dauphinée, S., Williams, J.I., Gayton, D.: Measuring Balance in the Elderly: Preliminary Development of an Instrument. Physiotherapy Canada 41(6), 304-311 (1989)

5. Betker, A.L., Szturm, T., Moussavi, Z.K., Nett, C.: Video game-based exercises for balance rehabilitation: a single-subject design. Archives of physical medicine and rehabilitation 87(8), 1141-9 (2006). https://doi.org/10.1016/j.apmr.2006.04.010

6. Bhuiyan, M., Picking, R.: Gesture-controlled user interfaces, what have we done and what's next. Proceed-ings of the 5th Collaborative Research Symposium on Security, E-Learning, Internet and Networking pp. 59-69 (2009)

7. Brito, C.M., Jacob, J., Nóbrega, R., Santos, A.M.: Balance Assessment in Fall-Prevention Exergames. In: Proceedings of the 17th International ACM SIGACCESS Conference on Computers' Accessibility. pp. 439-440 (2015). https://doi.org/10.1145/2700648.2811342

8. Campbell, A.J., Robertson, M.C.: Otago Exercise Programme to Prevent Falls in Older Adults (2003)

9. Clark, R.a., Bryant, A.L., Pua, Y., McCrory, P., Bennell, K., Hunt, M.: Validity and reliability of the Nintendo Wii Balance Board for assessment of standing balance. Gait and Posture 31, 307-310 (2010). https://doi.org/10.1016/j.gaitpost.2009.11.012

10. Davies, T.C., Deacon, M., Singh, J., Holly, Z., Taylor, L., Mathieson, S., Parsons, J.: Developing Wii Balance Games to Increase Balance: A Multi-Disciplinary Approach. International Journal of Virtual Worlds and Human Computer Interaction 1 (2013). https://doi.org/10.11159/vwhci.2013.002

11. Deutsch, J.E., Borbely, M., Filler, J., Huhn, K., Guarrera-Bowlby, P.: Use of a low-cost, commercially available gaming console (Wii) for rehabilitation of an adolescent with cerebral palsy. Physical therapy 88(10), 1196-1207 (2008). https://doi.org/10.2522/ptj.20080062

12. Duarte, M., Freitas, S.M.S.F.: Revisão sobre Posturografia Baseada em Plataforma de Força para Avaliação do Equilíbrio. Revista Brasileira de Fisioterapia 14(3), 183-192 (2010)

13. Eng, J., Dawson, A., Pang, M.: Fitness and Mobility Exercise Program: A community-based Group Exercise Program for People Living with Stroke (2006)

14. Geurts, L., Vanden Abeele, V., Husson, J., Windey, F., Van Overveldt, M., Annema, J.H., Desmet, S.: Digital games for physical therapy: fulfilling the need for calibration and adaptation. Design pp. 117-124 (2011)

15. González, A., Hayashibe, M., Fraisse, P.: Estimation of the Center of Mass with Kinect and Wii balance board. International Conference on Intelligent Robots and Systems pp. 1023-1028 (2012) 
16. Hile, E.S., Brach, J.S., Perera, S., Wert, D.M., VanSwearingen, J.M., Studenski, S.A.: Interpreting the need for initial support to perform tandem stance tests of balance. Physical therapy 92(10), 1316-28 (Oct 2012). https://doi.org/10.2522/ptj.20110283

17. Jacob, J., Nóbrega, R., Coelho, A., Rodrigues, R.: Adaptivity and safety in location-based games. In: 2017 9th International Conference on Virtual Worlds and Games for Serious Applications (VS-Games). pp. 173-174 (Sep 2017). https://doi.org/10.1109/VS-GAMES.2017.8056592

18. Jacob, J., Lopes, A., Nóbrega, R., Rodrigues, R., Coelho, A.: Player adaptivity and safety in location-based games. In: Cheok, A.D., Inami, M., Romão, T. (eds.) Advances in Computer Entertainment Technology. pp. 219-238. Springer International Publishing, Cham (2018)

19. Jacob, J., Lopes, A., Nóbrega, R., Rodrigues, R., Coelho, A.: Towards player adaptivity in mobile exergames. In: Cheok, A.D., Inami, M., Romão, T. (eds.) Advances in Computer Entertainment Technology. pp. 278-292. Springer International Publishing, Cham (2018)

20. Kennedy, M.W., Schmiedeler, J.P., Crowell, C.R., Villano, M., Striegel, A.D., Kuitse, J.: Enhanced feedback in balance rehabilitation using the Nintendo Wii balance board. 2011 IEEE 13th International Conference on e-Health Networking, Applications and Services, HEALTHCOM 2011 pp. 162-168 (2011)

21. Lange, B.S., Flynn, S.M., Chang, C.Y., Ahmed, A., Geng, Y., Utsav, K., Xu, M., Seok, D., Cheng, S., Rizzo, a.a.: Development of an interactive rehabilitation game using the Nintendo WiiFitTM Balance Board for people with neurological injury. Intl Conf. Disability, Virtual Reality $\{\&\}$ Associated Technologies pp. 249-254 (2010)

22. Langley, F.A., Mackintosh, S.F.H., Applsc, B.: Functional Balance Assessment of Older Community Dwelling Adults : A Systematic Review of the Literature. The Internet Journal of Allied Health Sciences and Practice 5(4), 1-11 (2007)

23. Mekler, E.D., Bopp, J.A., Tuch, A.N., Opwis, K.: A systematic review of quantitative studies on the enjoyment of digital entertainment games. Proceedings of the 32nd annual ACM conference on Human factors in computing systems - CHI '14 pp. 927-936 (2014). https://doi.org/10.1145/2556288.2557078

24. Mellecker, R., Lyons, E.J., Baranowski, T.: Disentangling Fun and Enjoyment in Exergames Using an Expanded Design, Play, Experience Framework: A Narrative Review. Games for health journal 2(3), 142-149 (Jun 2013). https://doi.org/10.1089/g4h.2013.0022

25. Melo, M.C.P.A.: The Impact of a Specific Home Based Exercise Programme on Fal Risk Factors in Older Portuguese People. Ph.D. thesis, University of Brighton (2008)

26. Mitty, E., Flores, S.: Fall Prevention in Assisted Living: Assesment and Strategies. Geriatric Nursing 28(6), 349-357 (2007)

27. Nawaz, A., Skjæ ret, N., Ystmark, K., Helbostad, J.L., Vereijken, B., Svanæ s, D.: Assessing seniors' user experience (UX) of exergames for balance training. In: Proceedings of the 8th Nordic Conference on Human-Computer Interaction Fun, Fast, Foundational - NordiCHI '14. pp. 578-587. New York, New York, USA (2014). https://doi.org/10.1145/2639189.2639235

28. Nicholson, V.P., McKean, M., Lowe, J., Fawcett, C., Burkett, B.: Six weeks of unsupervised Nintendo Wii Fit gaming is effective at improving balance in independent older adults. Journal of Aging and Physical Activity 23(1), 153-158 (2015). https://doi.org/10.1123/JAPA.2013-0148 
29. Nielsen, M., Störring, M.: A procedure for developing intuitive and ergonomic gesture interfaces for HCI. In The 5th Int. Workshop on Gesture and Sign Language based Human Computer Interaction pp. 1-12 (2003)

30. Pajala, S., Era, P., Koskenvuo, M., Kaprio, J., Törmäkangas, T., Rantanen, T.: Force Platform Balance Measures as Predictors of Indoor and Outdoor Falls in Community-Dwelling Women Aged 63-76 Years. The Journals of Gerontology Series A: Biological Sciences and Medical Sciences 63(2), 171-178 (Feb 2008). https://doi.org/10.1093/gerona/63.2.171

31. Pinto, D., Costa, J., Nóbrega, R., da Silva, H., Coelho, A.: Graphical simulation of clinical scenarios for medical training. In: 2018 International Conference on Graphics and Interaction (ICGI). pp. 1-8 (Nov 2018). https://doi.org/10.1109/ITCGI.2018.8602866

32. Podsiadlo, D., Richardson, S.: The Timed "Up \& Go": A Test of Basic Functional Mobility for Frail Elderly Persons. Journal of the American Geriatrics Society 39, 142-148 (1991)

33. Rose, D.J., Lucchese, N., Wiersma, L.D.: Development of a multidimensional balance scale for use with functionally independent older adults. Archives of physical medicine and rehabilitation 87(11), 1478-85 (Nov 2006). https://doi.org/10.1016/j.apmr.2006.07.263

34. Santos, A., Guimares, V., Matos, N., Cevada, J., Ferreira, C., Sousa, I.: Multi-sensor exercise-based interactive games for fall prevention and rehabilitation. In: 2015 9th International Conference on Pervasive Computing Technologies for Healthcare (PervasiveHealth). pp. 65-71 (May 2015). https://doi.org/10.4108/icst.pervasivehealth.2015.259115

35. Tyson, S.: Brunel Balance Assessment (BBA). University of Salford (2004)

36. Uzor, S., Baillie, L.: Investigating the long-term use of exergames in the home with elderly fallers. Proceedings of the 32nd annual ACM conference on Human factors in computing systems - CHI '14 pp. 2813-2822 (2014). https://doi.org/10.1145/2556288.2557160

37. Vines, J., Pritchard, G., Wright, P., Olivier, P.: An Age-Old Problem : Examining the Discourses of Ageing in HCI and Strategies for Future Research. TOCHI 22(1), 1-27 (2015). https://doi.org/10.1017/S0269888900008122

38. Zaczynski, M., Whitehead, A.D.: Establishing design guidelines in interactive exercise gaming. In: Proceedings of the 32nd annual ACM conference on Human factors in computing systems - CHI '14. pp. 1875-1884 (2014). https://doi.org/10.1145/2556288.2557329 